Dr. Clutterbuck mentioned the following case, to show the difficulties sometimes of arriving at pathological conclusions, and the little use of medical aid in some instances. He was consulted, some months since, by a female, thirty-five years of age, of full habit, and indolent mode of life, who suffered from a chronic affection of the liver, and complained of uneasiness at the epigastrium, and disorder of the stomach. As the symptoms were active she was bled twice, to the extent of six ounces, with great benefit, and she lost all symptoms of disease. She came again to him about a fortnight since, complaining of pain in the epigastrium, deficient appetite, and a degree of sleeplessness. He recommended mild aperients, to be followed by the infusion of gentian. He saw her while under the influence of this medicine, but the symptoms remained the same. As bleeding had relieved her before, and as the system did not want power, he allowed four ounces to be taken, the blood being highly buffed and cupped; but the symptoms changed not. The pulse was not at all weak; the tongue but very slightly coated; the skin not preternaturally hot; the surface not febrile; the uneasiness at the epigastrium, and the restlessness, continued. His attention was now called to some blotches of purpura on the legs, arms, and trunk. The pulse was strong, rather than otherwise; and if there had been any pain present, he would have recommended further abstraction of blood. He was struck with the opposite character of the symptoms; he expected to find much more debility, and a weaker pulse. He knew not how to act. He did not, therefore, proceed actively, but employed lemon-juice to as great an extent as could be borne. The ecchymoses, however, spread, and inclined to hæmorrhage; the saliva, urine, and fæces became bloody, and she menstruated dark-coloured blood. She breathed laboriously, from want of power to expand the chest. She grew weaker, and her pulse began to fail ; she complained of oppression, not pain, about the head, and confused vision. Tonics and stimulants were now employed, but had no effect on the disease, and the patient sank in two or three days. The post-mortem examination threw no light on the case ; and, after dissection, he was as much in the dark as before, regarding the pathology of the affection. Here was a case in which the heart and large vessels were acting with proper and natural energy, while the capillaries allowed the escape of blood. He understood neither the pathology nor the treatment of such a case. Oil of turpentine, in small doses, had been recommended in one of the journals, in cases of purpura, but in this instance there was no time to use that remedy.

\section{CHOREA, WITH FATAL TERMINATION.}

\section{To the Editor of The LANCET.}

SIR :-In relation to a case of chorea published in The LA NCET of August 19, I wish to mention one which arose from uncommon causes, where the patient came under my care some weeks before the symptoms of chorea showed themselves; and I trust that the physician under whose care the patient afterwards was placed will mention any other matter appertaining to the subject.

A girl, about thirteen years of age, came to the Nottingham Hospital with a fungous excrescence protruding from beneath the lower portion of the thumb-nail (of which hand I know not), and which had progressed rather rapidly, attended with uneasiness. After touching the fungus with solution of the nitrate of silver four or five times, and twice with potassa fusa, it healed. To my astonishment, within a month afterwards she was admitted into the house suffering under the most unequivocal symptoms of chorea, which were assuming daily a worse aspect. During the last three or four days before she died, she passed her fæces and urine involuntarily, had delirium, sleepless nights, and bed-sores, probably from friction, though she was kept.as clean as possible. The treatment consisted in the administration of good doses of carbonate of iron and quinine. Towards the last, calomel, \&c., and blisters, and other derivatives, were employed. I fancy leeches were applied behind the ears, when the disease was getting obstinate. The postmortem dissection exposed to view serous effusion, and a somewhat softened state of the brain. I am, Sir, your obedient servant,

Brompton, Sept., 1837.

R. DAY.

THE IODURETTED-I ODIDE OF POTASSIUM,

A DELICATE TEST FOR BICHLORIDE OF MERCURY.

To the Editor of THE LANCET.

SIR:-As every additional test increases the certainty with which we may decide upon the presence of a poison in a suspected liquid, the fact that the liquor potassii iodidi compositus of the New Pharmacopoia will detect one grain of corrosive sublimate in fourteen thousand grains of water, may not prove unacceptable to the readers of your valuable periodical.

My mode of operating is, if the bichloride of mercury be united with gruel, soup, or coffee, to dilute the suspected liquid with water slightly acidulated by hydrochloric (muriatic) acid, and then filter it through animal charcoal. Placing my test-tube upon a sheet of white paper, I carefully drop 
into the liquid I wish to examine some of the ioduretted solution, allowing it to obey the laws of gravity, and fall to the bottom of the glass. Viewing it by the light reflected from the paper, we shall perceive, if it contains corrosive sublimate, a light clouding over the surface of the brown fluid at the bottom, which gradually increases, and, contrasted with the white paper, is seen to be distinctly a reddish-brown powder, which I am led to suspect is a tritiodide of mercury.

It cannot be too generally known to medical practitioners that the potassii iodidi, kept by the druggists in general, is largely adulterated with the bicarbonate of potash. Twelve samples obtained from as many respectable shops, I found to contain from fifteen to fifty per cent. of this salt. I am, Sir, your obedient servant,

32, Newgate-gtreet,

Oct. 24th, 1837.

\section{CREOSOTE IN CHOLERA.}

To the Editor of THE LANCET.

SIR:-Having seen a statement of the efficacy of creosote in a case of cholera morbus, in The LanceT of September 16th, I was induced to use it in the subjoined case, the result of which induces me to lay it before the profession through the same medium. I may add, that although it has been recommended in the disease alluded to, by experienced practitioners, it has not been generally adopted, and I would, therefore, suggest the publication of such cases wherein it has been, or may be given, with a view to ascertain completely its efficacy in the disease.

Case.-Peter M'Cannon, an Irish reaper, about 40 years of age, was attacked, on the morning of the 8th inst., at 4 o'clock. At 10 I found him affected with vomiting, purging, cramps, cold extremities, and weak voice, the pulse being scarcely perceptible, and the countenance shrunk. His symp. toms were of the worst description. 1 immediately proceeded to use calomel and opinm with the other auxiliary remedies, and persevered in their application for two hours without effecting any change. It then occurred to me to add a drop of creosote to each dose of calomel and opium, which I immediately prepared to the extent of three doses. The first dose was given at half-past twelve, but was soon rejected; the second dose shortly afterwards, which was retained for half an hour; and the third dose, given soon after the rejection of the former, was kept for three hours. I have only to add, that by three o'clock reaction had taken place, und the case progressed rapidly and favourably from that time. Thirst and costiveness were the prominent symptoms after that period. I am, Sir, your obedient servant,

Morpeth, Northumberland, Oct. 24, 1837.

\section{ON CONTUSION AND CONCUSSION OF THE BRAIN.}

ThE numbers of the "Archives Générales de Medecine," for May, July, and Septem. ber, contain a well-written memoir on the immediate signs of contusion of the brain, by M. Boinet, interne at the Hotel Dieu, of which the following is a resumé.

The symptoms of contusion of the brain have been hitherto overlooked by the best surgeons, and in the most recent treatises on the art of surgery; or, if mentioned, they have been almost invariably confounded with the symptoms resulting from compres. sion or concussion of that organ. M. Sanson, of the Hotel Dieu, was one of the first to point out that contusion of the brain is immediately followed by a certain set of phenomena, which are pathognomonic of the lesion, and which will generally enable us to distinguish it from concussion and compression.

Anatomically considered, contusion of the brain may exist in three forms or degrees. The first is characterised by rupture of the vessels passing from the brain to the dura mater, or from the latter to the cranium, without apparent injury of the cerebral substance. In the second degree we have, in addition, a superficial lesion of the cerebral substance; the grey matter is ecchymosed, but not softened. These two forms admit of cure. In the third degree the substance of the brain is profoundly disorganised; its tissue, in one or more points, is reduced to a detritus in which the grey and white substances are confounded together; the mem. branes are lacerated, and more or less effusion of blood takes place.

The pathognomonic signs of contusion of the brain are, according to M. Sanson, more or less contracture of the limbs, a constant agitation of the whole body, and loss of consciousness, without the stertorons respiration, occurring immediately after the receipt of the injury. In slight eases, however, the contraction of one pupil, or of an eyelid, the spasmodic movement of the lips, or of a single muscle, \&c., and difficulty of pronouncing certain words, are the only signs of contusion of the brain which present themselves. As contusion of the brain is almost always produced by external violence inflicted about the region of the head, the symptoms will vary according to the multiplicity or extent of the lesions which result. 